\title{
Green Tea Treats and Prevents Infectious Diseases
}

\author{
Dr. Anil Batta*
}

Professor and Head, Department of medical biochemistry Govt. Medical College, Amritsar

\author{
DOI: $\underline{10.36348 / \text { sijb.2020.v03i10.002 }}$
}

| Received: 30.09 .2020 | Accepted: 13.10.2020 | Published: 18.10 .2020

*Corresponding author: Dr. Anil Batta

\section{Abstract}

Green tea is one of the most popular drinks consumed worldwide. Produced mainly in Asian countries from the leaves of the Camellia sinensis plant, the potential health benefits have been widely studied. Recently, researchers have studied the ability of green tea to eradicate infectious agents and the ability to actually prevent infections. The important components in green tea that show antimicrobial properties are the catechins. The four main catechins that occur in green tea are (-)epicatechin (EC), (-)-epicatechin-3-gallate (ECG), (-)-epigallocatechin (EGC), and (-)-epigallocatechin-3-gallate (EGCG). Of these catechins, EGCG and EGC are found in the highest amounts in green tea and has been the subject of most of the studies. These catechins have been shown to demonstrate a variety of antimicrobial properties, both to organisms affected and in mechanisms used. Consumption of green tea has been shown to distribute these compounds and/or their metabolites throughout the body, which allows for not only the possibility of treatment of infections but also the prevention of infections.

Keywords: Green Tea Treats and Prevents.

Copyright $\odot \mathbf{2 0 2 0}$ The Author(s): This is an open-access article distributed under the terms of the Creative Commons Attribution 4.0 International License (CC BY-NC 4.0) which permits unrestricted use, distribution, and reproduction in any medium for non-commercial use provided the original author and source are credited.

\section{INTRODUCTION}

The prevalence of overweight and obesity and associated metabolic complications and related morbidity has increased dramatically in the past two decades [1]. Obesity or body mass index (BMI) $>30$ $\mathrm{kg} / \mathrm{m} 2$ is a disease that affects more than $30 \%$ of United States adults, with a higher incidence among women. It is expected that one in three children born in the early current century will develop diabetes associated with obesity [1,2]. In addition to diabetes, obesity is a major risk factor for cardiovascular diseases, several forms of cancer (such as breast, colon, and prostate), pulmonary, osteoarticular and metabolic diseases [3-8]. It also accounts for $5-7 \%$ of the national health expenditure in the United States [9]. Thus, obesity is considered a major threat to the public's health. Diet and behavioral modification programs for weight loss and the prevention of weight regain aims to reduce energy intake and increase energy expenditure. However, the ineffectiveness of most approaches is seen in the fact that the prevalence of obesity is at an all-time high and that weight regain is common [10]. Over the past two decades, chemicals derived from plants and known as "phytochemicals" have gained the interest of public and scientific communities for their role in maintaining health and preventing disease. Polyphenols derived from many components of the human diet are among the leading phytochemicals, and some of their potential preventive and therapeutic properties have been studied extensively. In this review, the polyphenols with potential efficacy to modulate obesity and associated disorders are briefly discussed. Tea is one of the most popular beverages and is widely consumed throughout the world. Several forms of tea including green, black, oolong and white teas are prepared from the harvest of leaves from the Camellia sinensis plant. The teas differ by their processing and bioactive chemical contents. Green tea has been well-investigated and is recognized by ancient Chinese medicine as having many significant health effects on humans. In recent years, the health benefit effects of green tea are mainly attributed to high concentrations of polyphenols, which are collectively called catechins. Green tea contains five major catechins including: catechin, epicatechin, epicatechin gallate, epigallocatechin, and epigallocatechin gallate (EGCG); the latter comprises more than $40 \%$ of the total polyphenol mixture of green tea catechins. Epidemiological, experimental, and clinical studies have suggested several beneficial effects from consuming green tea: antioxidant and anti-inflammatory activities, cancer and cardiovascular disease prevention properties, and anti-obesity. The anti-obesity effect of green tea is mainly attributed to catechins, in particular EGCG, which exhibits its anti-obesity effects through several mechanisms including suppression of adipocyte 
differentiation and proliferation, inhibition of fat absorption from the gut, and suppression of catechol-omethyl transferase (COMT), an enzyme that inhibits fatty acid oxidation in brown adipose tissue.

\section{Composition of green tea}

The components in green tea that are the most medically relevant are the polyphenols. The most pertinent polyphenols are the flavonoids; and the most pertinent flavonoids are the catechins. The catechins comprise $80-90 \%$ of the flavonoids and around $40 \%$ of the water-soluble solids in green tea. Green tea contains more catechins than the other teas, mainly because of the way it is processed after harvesting. The amount of catechins in green tea can also be affected by where the tea is grown, the growth conditions, when it is harvested, how the leaves are processed, and the brewing temperature and length of time of brewing. These factors lead to a huge variation in catechin content among the varieties and brands of green tea consumed [35-45]. The four main catechins found in green tea are (-)-epicatechin (EC), (-)-epigallocatechin (EGC), (-)-epicatechin-3-gallate (ECG), and (-)epigallocatechin-3-gallate (EGCG). The most abundant catechin is EGCG ( 60\%), and the next most abundant is $\mathrm{EGC}(\sim 20 \%)$, then ECG $(\sim 14 \%)$, and EC $(\sim 6 \%)$. EGCG is the most studied in association with health, but EGC and ECG have been studied as well. As mentioned above, there can be a wide variation in the amount of catechins in any particular green tea beverage, although standardized extracts are available for use as supplements [7, 46, 47]. In order to be effective in the body these catechins need to be bioavailable after consumption. Once in the body, the catechins undergo metabolic processing in the liver and small intestine and colon. This processing produces glucuronide and sulfate conjugates or methyl epicatechins.

Table -1: Amount of ecgc in blood plasma after a single dose

\begin{tabular}{|l|l|}
\hline EGCG dose (mg) & (ng/ml) \\
\hline 200 & 73.7 \\
\hline 400 & 111.8 \\
\hline 600 & 169.1 \\
\hline 800 & 438.5 \\
\hline 400 & 137.6 \\
\hline 800 & 234.9 \\
\hline
\end{tabular}

\section{Anti-microbial properties}

The antimicrobial effects of green tea catechins (GTCs) on microorganisms have been studied for many years. Green tea has been shown to combat these organisms in various ways, directly and indirectly, and has been shown to work synergistically with some antibiotic agents. Other known health benefits of green tea such as the anti-inflammatory and antioxidant effects may also contribute to the antimicrobial effects. Studies conducted on Escherichia coli found that exposure to green tea polyphenols (GTPs) resulted in major gene expression changes for 17 genes, with upregulation occurring in nine genes and downregulation in eight genes [75-77]. Table 3 shows a summary of the antimicrobial effects of green tea on bacteria.

\section{Effects on microbial cell membrane}

One of the major properties of GTCs is the ability to bind to bacterial cell membranes. This binding can lead to interference in various bacterial processes and can damage the cell membrane resulting in increased permeability and leading to cell lysis. Because EGCG is negatively charged it can combine with the positively charged bacterial cell membrane, especially in gram positive bacteria. The lipopolysaccharide (LPS) on the outer membrane of gram negative bacteria makes them more resistant to binding by GTCs $[53,63,64,66]$. Studies with E. coli and Pseudomonas aeruginosa have shown that EGCG binding to the bacterial cell membrane can result in generation of $\mathrm{H}_{2} \mathrm{O}_{2}$ which is involved in damage to the cell membrane [63, 74]. Studies with Staphylococcus aureus have shown that this assault on the cell membrane causes a major cell wall stress response, resulting in upregulation of peptidoglycan biosynthesis genes and an alteration in cell wall structure. In methicillin-resistant Staphylococcus aureus (MRSA) strains, this change in peptidoglycan biosynthesis genes results in the production of PBP2 (penicillin-binding protein 2), which is what confers resistance to $\beta$-lactam drugs. Production of PBP 2 is also inhibited by EGCG $[64,78,79]$. An important result of green tea binding is the loss of bacterial ability to bind to host cells. Studies using human and mammalian cells lines have shown that various bacteria such as Fusobacterium nucleatum, Staphylococcus epidermidis, and Helicobacter pylori have significantly decreased adherence to these cells $[66,67,80]$. Other important results are the loss of the ability for quorum sensing and biofilm formation of $\mathrm{P}$. aeruginosa, $\mathrm{F}$. nucleatum, and Streptococcus mutants $[66,68,81]$. Damage to the cell membrane also results in loss of function to transmembrane transporter proteins which are responsible for secretion of toxins and efflux of substances such as antimicrobial agents $[53,65,69,70]$.

Table- 2: Amount of egc in 24 hrs urine collection

\begin{tabular}{|l|c|}
\hline EGC dose (mg) & Amount in 24 hour urine (mg) \\
\hline & \\
\hline 37 & 0.4 \\
\hline 74 & 1.4 \\
\hline 111 & 3.5 \\
\hline 148 & 3.7 \\
\hline 102 & $\sim 3.0$ \\
\hline 82 & $\sim 3.0$ \\
\hline 102 & $\sim 3.0$ \\
\hline 204 & $\sim 4.0$ \\
\hline 306 & $\sim 4.8$ \\
\hline
\end{tabular}




\section{Effects of bacterial cell membrane}

There are a wide variety of other effects that GTCs have on bacterial functions. An important one which can affect most bacteria is the ability of GTCs to inhibit bacterial fatty acid biosynthesis by inhibiting enzymes involved in the biosynthetic pathway. Because this is an essential pathway for most bacteria, researchers are looking at targeting this pathway in antimicrobial drug development. Fatty acids are important for building cell membranes, as an energy source, and are involved in the production of toxic bacterial metabolites [53,73]. Another target is the folate biosynthesis pathway. The enzyme dihydrofolate reductase (DHFR) is essential in this pathway, and is known to be a target for certain sulfa drugs. EGCG has also been shown to inhibit DHFR activity [53, 82]. Other important effects against enzymes include inhibition of bacterial DNA gyrase, inhibition of bacterial ATP synthase activity, and inhibition of bacterial protein tyrosine phosphatase and cysteine proteases $[53,71,83]$.

\section{Synergism}

Since GTCs are known to have antimicrobial action, researchers have begun assessing the potential synergism of these catechins with other known antimicrobial agents. Green tea catechins have now been shown to act in synergy with imipenem against MRSA; with metronidazole against Porphyromonas gingivalis; with azithromycin, cefepime, ciprofloxacin, chloramphenicol, doxycycline, erythromycin, nalidixic acid, piperacillin, or tobramycin against E. coli; with ampicillin, Cefalotin, doxycycline, erythromycin, penicillin, or tetracycline against Enterobacter aerogenes; with chloramphenicol or tetracycline against Pseudomonas aeruginosa; and with aztreonam, ceftazidime, ciprofloxacin, gentamicin, meropenem, or tetracycline against Acinetobacter baumannii.

\section{Effects on other microorganisms}

Green tea catechins have also been shown to be effective against a number of viruses, parasites, fungi, and even prions. The main antiviral effects include inhibiting the virus from binding to and entering host cells (adenovirus, enterovirus, HBV, $\mathrm{HCV}$, HIV, HSV, influenza, and rotavirus); inhibiting viral RNA and DNA synthesis and viral gene transcription (enterovirus, EBV, HBV, HCV, and HIV); and destroying and functionally altering various viral molecules (adenovirus, HSV, and influenza) [64, 90 96]. Studies performed with adult healthcare workers to determine if green tea supplements could prevent infection with viruses causing influenza showed significantly fewer instances of influenza symptoms and a reduced incidence of laboratory-confirmed influenza cases versus the control group [97]. The main effect of GTCs on various parasite infections is a decrease in parasite numbers and growth. Other effects noted were fragmentation of parasite DNA and reduced fatty acid synthesis in the parasites. Studies with parasites include Plasmodium falciparum, Babesia spp., Trypanosoma brucei, Trypanosoma cruzi, and Leishmania braziliensis [98-102]. Fungi that have been affected by GTCs include Aspergillus Candida spp., Penicillium sp., Microsporum canis, Trichophyton mentagrophytes, and Trichophyton rubrum. Research testing for synergistic effects found that EGCG showed synergism with amphotericin B, fluconazole, and miconazole in Candida spp.; and in Candida tropicalis strains that were resistant to fluconazole, EGCG, and fluconazole together induced apoptosis in the yeast cells [64, 103-107]. Prions are proteins that are considered to be infective agents because the abnormally structured ( $\beta$-sheet) forms are able to induce normally structured ( $\alpha$-helix) forms to change shape. In the abnormal shape, protein function is lost and protein aggregation occurs in cells. Unlike other infectious agents, prions cannot be destroyed using autoclaving; the proteins have to be degraded to be noninfectious. Research using yeast cells found that EGCG could inhibit the $\beta$-sheet prions from changing the $\alpha$-helical forms and could induce reversal of the $\beta$ sheet forms back to $\alpha$-helical forms [108].

Table-3: Antimicrobial effect of green tea

\begin{tabular}{|l|l|}
\hline Organism & \multicolumn{1}{c|}{ Effects } \\
\hline $\begin{array}{l}\text { Cell Membrane } \\
\text { Associate Effects }\end{array}$ & $\begin{array}{l}\text { Bacterial Cell Functions Inhibits bacterial Inhibits ability of bacteria to bind to host cells } \\
\text { Inhibits ability of bacteria to form biofilms Disrupts bacterial quorum sensing Interferes with } \\
\text { bacterial membrane transporters }\end{array}$ \\
\hline $\begin{array}{l}\text { Bacterial Cell } \\
\text { Function }\end{array}$ & $\begin{array}{l}\text { DNA gyrase effects Reduces bacterial } \mathrm{H}_{2} \mathrm{~S} \text { production Inhibits bacterial hemolytic action } \\
\text { Inhibition of bacterial DHFR enzyme Inhibits bacterial fatty acid synthesis enzymes Increases } \\
\text { bacterial internal ROS levels }\end{array}$ \\
\hline
\end{tabular}

\section{Prevention of infection}

Since it has been shown that GTCs have multiple types of antimicrobial abilities against so many organisms, it would be expected that green tea catechins could also prevent infections. One study was mentioned previously describing how green tea reduced the number of colds and influenza incidents. Another study involving adults showed that consuming green tea supplements twice daily for 3 months resulted in $32 \%$ fewer instances of cold or influenza symptoms and nearly $23 \%$ fewer illnesses of 2 or more days duration [110]. A study involving children found that, in schoolaged children who consumed green tea on a regular basis, the number of incidents of influenza A or B was inversely associated with the number of cups of green tea consumed per day or per week [111]. Another study 
with Japanese nursey school children who gargled with green tea (or placebos) at least once each day found that there were up to 3 times fewer instances of illnesses with fevers in the green tea gargling group [112]. Two other studies with adults found that gargling with a green tea extract (GTE) solution resulted in at least half as many cases of influenza in the GTE gargling groups compared with the control groups.

\section{CONCLUSION}

The research into the effects of green tea on human health has shown that it can be an important dietary factor in the prevention and treatment of various diseases such as arthritis, cancer, CVD, diabetes and obesity, infections, and in neurologic and oral health. Studies that were originally performed in animals and cell lines have become more frequently performed using humans. This type of research is vital if we are to fully discover what benefits GTCs can have in health issues. The more researchers that become involved in this, the clearer the answers. The studies on antimicrobial effects are providing very promising data, especially if GTCs prove to have synergistic abilities with many of the currently used antimicrobial agents and perhaps with drugs used to treat other diseases. The emergence of various multidrug-resistant bacteria, along with a dearth of effective antimicrobial drugs, makes the potential of green tea an extremely timely issue. There are also many areas across the globe where the cost of drugs is currently beyond the earning power of most of the population. Green tea is relatively inexpensive and fairly easy to obtain for most people. It could prove to be an answer for improving health on a global scale.

\section{REFERENCES}

1. World Health Organization. (2014). "World Health Organization,"

2. U.S. Department of Health and Human Services Centers for Disease Control and Prevention. (2013). "Antibiotic resistance threats in the United States"

3. Stone, P. W. (2009). Economic burden of healthcare-associated infections: an American perspective. Expert review of pharmacoeconomics \& outcomes research, 9(5), 417-422.

4. Smith, R., \& Coast, J. (2013). The true cost of antimicrobial resistance. Bmj, 346, f1493.

5. Botten, D., Fugallo, G., Fraternali, F., \& Molteni, C. (2015). Structural properties of green tea catechins. The Journal of Physical Chemistry $B, 119(40), 12860-12867$.

6. Gupta, D. A., Bhaskar, D. J., Gupta, R. K., Karim, B., Jain, A., \& Dalai, D. R. (2014). Green tea: A review on its natural anti-oxidant therapy and cariostatic benefits. Biol. Sci. Pharm. Res, 2, 8-12.

7. Jigisha, A., Nishant, R., Navin, K., \& Pankaj, G. (2012). Green tea: a magical herb with miraculous outcomes. Int. Res. J. Pharm, 3(5), 139-148.

8. Hayat, K., Iqbal, H., Malik, U., Bilal, U., \& Mushtaq, S. (2015). Tea and its consumption: benefits and risks. Critical reviews in food science and nutrition, 55(7), 939-954.

9. Crew, K. D., Ho, K. A., Brown, P., Greenlee, H., Bevers, T. B., Arun, B., \& Rimawi, M. (2015). Effects of a green tea extract, Polyphenon E, on systemic biomarkers of growth factor signalling in women with hormone receptor- negative breast cancer. Journal of Human Nutrition and Dietetics, 28(3), 272-282.

10. Li, M. J., Yin, Y. C., Wang, J., \& Jiang, Y. F. (2014). Green tea compounds in breast cancer prevention and treatment. World journal of clinical oncology, 5(3), 520 . 